\title{
Accuracy-Privacy Tradeoffs for Two-Party Differentially Private Protocols
}

\author{
Vipul Goyal ${ }^{1, \star}$, Ilya Mironov ${ }^{2}$, Omkant Pandey ${ }^{3, \star}$, and Amit Sahai ${ }^{4, \star \star}$ \\ 1 Microsoft Research India \\ 2 Microsoft Research Silicon Valley \\ 3 The University of Texas at Austin \\ 4 University of California Los Angeles
}

\begin{abstract}
Differential privacy (DP) is a well-studied notion of privacy that is generally achieved by randomizing outputs to preserve the privacy of the input records. A central problem in differential privacy is how much accuracy must be lost in order to preserve input privacy?

Our work obtains general upper bounds on accuracy for differentially private two-party protocols computing any Boolean function. Our bounds are independent of the number of rounds and the communication complexity of the protocol, and hold with respect to computationally unbounded parties. At the heart of our results is a new general geometric technique for obtaining non-trivial accuracy bounds for any Boolean functionality.

We show that for any Boolean function, there is a constant accuracy gap between the accuracy that is possible in the client-server setting and the accuracy that is possible in the two-party setting. In particular, we show tight results on the accuracy that is achievable for the AND and XOR functions in the two-party setting, completely characterizing which accuracies are achievable for any given level of differential privacy.

Finally, we consider the situation if we relax the privacy requirement to computational differential privacy. We show that to achieve any noticeably better accuracy than what is possible for differentially private two-party protocols, it is essential that one-way functions exist.
\end{abstract}

\section{Introduction}

SFE and Differential Privacy. Secure function evaluation (SFE) is a fundamental concept in cryptography. Informally, SFE allows two parties to compute a joint function of their inputs without learning anything other than the

\footnotetext{
* Part of the work was done at Microsoft Research Silicon Valley.

** Research supported in part from a DARPA/ONR PROCEED award, NSF grants 1228984, 1136174, 1118096, 1065276, 0916574 and 0830803, a Xerox Faculty Research Award, a Google Faculty Research Award, an equipment grant from Intel, and an Okawa Foundation Research Grant. This material is based upon work supported by the Defense Advanced Research Projects Agency through the U.S. Office of Naval Research under Contract N00014-11-1-0389. The views expressed are those of the author and do not reflect the official policy or position of the Department of Defense, the National Science Foundation, or the U.S. Government.
}

R. Canetti and J.A. Garay (Eds.): CRYPTO 2013, Part I, LNCS 8042, pp. 298-315, 2013.

(C) International Association for Cryptologic Research 2013 
value of the function. An important research program in SFE is characterization of assumptions necessary for computing various classes of functionalities. For instance, an early result of Chor and Kushilevitz [7] established a zeroone law for Boolean functions in the information-theoretic model (against a computationally-unbounded passive adversary).

Differential privacy (DP) is a theoretically sound and practically important notion of privacy 1012 . In contrast with SFE (which places the "output correctness first and privacy second"), it limits information leaked through the output of the function (i.e., places "privacy first and output correctness second"). Differential privacy mechanisms work by randomizing the output to preserve the privacy of the input records. Thus the main question in differential privacy is quantitative rather than qualitative: How much accuracy must be lost in order to preserve input privacy?

The problem of characterizing accuracy of differentially private mechanisms is well-defined and very challenging even in the case of a single party's holding the input (the client-server setting). However if the input is distributed across several parties, output needs to be computed through an interactive protocol. Throughout the protocol, parties are restricted in how much information their messages should reveal about their input, and this would seem to degrade the quality of the output.

In other words, differential privacy gives a new set of restrictions on a protocol. Compared with the long line of research on feasibility and completeness of SFEs and MPCs for various functionalities in the semi-honest computationallyunbounded setting [3624/1/22 23], our understanding of the corresponding properties of differentially private protocols is remarkably incomplete.

The notion of differential privacy has been studied in the distributed setting, starting with the seminal work of Dwork and Nissim [13. In their work, there are multiple parties each holding a dataset as the input. The study of limitations on accuracy of distributed differentially private protocols was initiated in works of Beimel et al. 2] for the case of $n$ parties each holding its own input, followed up by Chan et al. [5], and of McGregor et al. 25] for the setting of two parties with $n$-bit inputs. The latter work considers several natural and constructed functionalities that exhibit a stark gap in accuracy that can be as large as $\Theta(n)$ between client-server and two-party protocols.

However, many questions remain. While we have several examples of functionalities for which there is an accuracy gap between the client-server setting and the distributed setting, does such a gap exist for any non-trivial functionality? How large must this gap be? Answering these questions for a large and natural class of functionalities in the two-party setting is the main focus of this work.

Boolean Functionalities. In this work, we focus on protocols that attempt to compute a Boolean function. While much work in differential privacy has focused on computing statistics, we note that computing Boolean functions has long been a motivating goal in differential privacy (e.g., answering questions "Does smoking cause cancer?" or "Do millionaires pay proportionally less in tax than their secretaries?"). Our goal is to obtain a characterization of which 
Boolean functions must suffer accuracy loss in the two-party setting, as well as lower bounds on how much accuracy loss is inherently needed. We note that our understanding of Boolean functions has been particularly (and perhaps surprisingly) weak: Before this work, even for computing simple Boolean gates, like AND and XOR, we did not understand whether any accuracy loss is essential to the two-party setting.

\subsection{Our Results}

Before we describe our results, we must define the notion of accuracy that we measure. Since our focus is on functions with Boolean output, there is only one natural choice for accuracy measure: the probability that the output is correct. We note that other metrics considered in the literature do not apply to the Boolean setting.

Now we discuss our setting in more detail. There are two parties Alice and Bob holding inputs $x$ and $y$ respectively and interested in computing a Boolean function $f(x, y)$. The protocol should be such that the differential privacy of each bit of $x$ as well as of $y$ should be preserved. We assume that Alice and Bob follow the protocol as specified, but keep a record of what transpired during the protocol (i.e., they are semi-honest in the cryptographic sense).

For a protocol to achieve accuracy $a$, it must be the case that for any possible inputs $(x, y)$ to the protocol, the protocol computes the correct output with probability at least $a$, over the coins of the protocol. We concentrate on the worst-case (over the parties' inputs) measure of accuracy as it is the most general type of guarantee for a randomized protocol, independent of distributional assumptions.

Informally speaking, the differential privacy (DP) constraint for Alice states that for any two inputs $x_{0}, x_{1}$ for Alice that differ only in one bit, and for any input $y$ for Bob, the following must hold: For every possible execution of the protocol, the resulting view $v$ of Bob must be such that the probability that $v$ arises on inputs $\left(x_{0}, y\right)$ is within a multiplicative factor of $e^{\epsilon}$ from the probability that $v$ arises on inputs $\left(x_{1}, y\right)$ (see Section 2 for the formal definition of differential privacy). Thus, no matter what Bob sees, he remains uncertain about the value of each bit of Alice's input even if he knows every other bit in her input. Here $\epsilon$ is the key privacy parameter. We will denote by $\lambda$ the value $e^{\epsilon}$. It is easy to see that in the client-server setting, it is always possible to achieve an accuracy of $\frac{\lambda}{1+\lambda}(=.5+\Theta(\epsilon)$ for $\epsilon \rightarrow 0)$.

Our work obtains general upper bounds on accuracy. Our bounds are independent of the number of rounds and the communication complexity of the protocol, and hold with respect to computationally unbounded parties. At the heart of our results is a new general geometric technique for obtaining non-trivial accuracy bounds for any Boolean functionality.

\footnotetext{
${ }^{1}$ Stronger notions of privacy are also interesting: for example, where symbols larger than bits, or the entire input of each party should be protected. However, since our focus is on obtaining lower bounds on error, we use the weaker notion of the privacy stated here, with respect to bits.
} 
General Boolean Functions. Our strategy to obtain results on two-party differentially private protocols for general Boolean functions begins by reducing the problem of obtaining upper bounds for general Boolean functions to specific, simple functions. We first note that Boolean functions where one party's input completely determines the output can, of course, be computed just as accurately in the two-party setting as in the client-server setting. We call such functions trivial, following works on classifying which Boolean functions have statisticallysecure two-party SFE protocols. We then show that the existence of an $\epsilon$-DP protocol with accuracy $a$ for any non-trivial function implies the existence of an $\epsilon$-DP protocol with accuracy $a$ for either the AND or XOR functionalities (defined below). Thus, if we can obtain upper bounds on accuracy for AND and XOR, we obtain upper bounds on accuracy for all non-trivial Boolean functions.

Computing an AND Gate. The AND functionality is as follows: Alice and Bob each hold a bit denoted by $x$ and $y$ respectively and are interested in computing the AND of the two bits. Given the output (and the protocol transcript), each input bit should remain private. Naively, the best differentially private protocol for this task is the randomized response protocol: each party individually perturbs its input and sends it out. The parties then compute the output based on the two input bits appearing in the protocol transcript. It is easy to see that the output and the protocol transcript still maintain privacy of each individual bit; moreover, both players' bits are released with maximal possible accuracy. The randomized response technique gives protocols for AND with accuracy $\frac{\lambda^{2}}{(1+\lambda)^{2}}$, which for $\lambda<1+\sqrt{2}$ is worse than a random guess.

We show that by augmenting the parties' outputs with one additional symbol, it is possible to improve on the naïve protocol. The new protocol can achieve an accuracy of $\frac{\lambda\left(\lambda^{2}+\lambda+2\right)}{(1+\lambda)^{3}}$. Moreover, we show that this accuracy is optimal for AND, even for protocols with any number of rounds and unbounded (finite) communication complexity. For $\epsilon \rightarrow 0$ and $\lambda \approx 1+\epsilon$ the protocol's advantage over a random guess is $\Theta(\epsilon)$, in line with the canonical protocol in the clientserver setting.

Computing an XOR Gate. The XOR functionality is defined analogously to the AND functionality above, except the XOR of the two input bits is to be computed. For the XOR case, the randomized response technique provides an accuracy of $\frac{1+\lambda^{2}}{(1+\lambda)^{2}}=.5+\Theta\left(\epsilon^{2}\right)$ for $\epsilon \rightarrow 0$. We show that this is, in fact, optimal for XOR.

Combining the results above, we establish the following: There does not exist any non-trivial Boolean functionality which can be computed with a differential private protocol in the two party setting with accuracy matching that of the clientserver setting. In fact, we obtain a separation between the level of accuracy obtainable in the client-server setting and the two-party setting for every nontrivial Boolean functionality, where the separation is tight in the case of AND and XOR, and for the XOR functionality is asymptotically significant. Our bounds are shown in Figure 1 . 


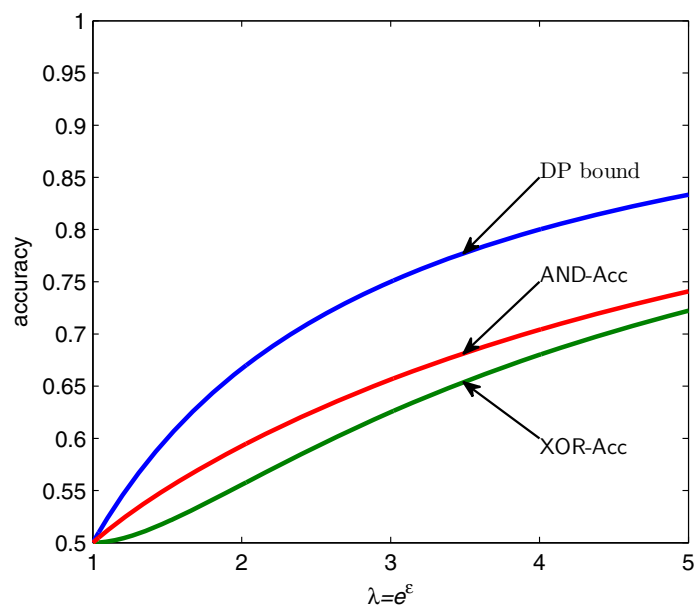

Fig. 1. Bounds on accuracy: for arbitrary Boolean functionalities (DP bound); for protocol-compatible $\epsilon$-DP AND and XOR. Every $\epsilon$-DP protocol for any non-trivial Boolean functionality must be subject to either the AND or XOR bound.

\section{Computational Differential Privacy: What Assumption Is Necessary?}

One option to restore accuracy in a distributed setting is to resort to a relaxed computational notion of differential privacy [26]. In computational differential privacy (CDP), we relax the privacy condition to require that no efficient adversary can predict any bit of the input with probability greater than $\frac{\lambda}{1+\lambda}$, even if the adversary knows all other bits. We ask the question: what computational assumptions are necessary for CDP to enable greater accuracy?

We show that to achieve any noticeably greater accuracy with CDP protocols than what is possible with DP protocols, one-way functions are required. We show this by presenting a more general result, showing that if one-way functions do not exist, then any CDP protocol must in fact also be a DP protocol.

When discussing CDP protocols, it is important to consider the relationship between CDP protocols and secure computation protocols from cryptography 3015 . The two notions answer essentially orthogonal problems of what and how:

- In (computationally) differentially private protocols, "privacy comes first". We would like to ensure privacy of each individual input and then with this constraint, would like to compute an accurate output (the what question).

- In secure computation protocols, "accuracy comes first". We would like to release an accurate output to the function we are computing and then with this constraint, would like to ensure privacy of inputs (the how question).

Nevertheless, it is immediate that general secure computation methods do give a way to achieve the same level of accuracy in CDP two-party protocols as in the client-server setting. To this end, a secure computation can be used to compute 
the algorithm that the server would perform on the joint input in the clientserver setting. However, general secure computation is essentially equivalent to secure Oblivious Transfer 21|19. It remains an important open question whether accuracy better than what is possible with DP protocols may be achievable based on assumptions weaker than the existence of secure Oblivious Transfer protocols.

\subsection{Our Techniques}

We present a new general geometric technique for bounding the accuracy of differentially private protocols. At a high level, our technique gives us a method for taking the truth table of a function $f$, a privacy parameter $\epsilon$, and an accuracy level $a$, and converting this into a linear program $P$. We prove that if there does exist an $\epsilon$-DP protocol for computing $f$ with accuracy $a$, then this linear program must have a solution. By analyzing this LP in the case of specific functions, we can show that no solution exists when $a$ is greater than a bound $a^{*}$. This proves that no $\epsilon$-DP protocol can exist with accuracy greater than $a^{*}$.

For simplicity, let us focus on protocols for Boolean functions where each party holds a single bit. To obtain our bounds, we first think of every possible "transcript" corresponding to some execution of the protocol. We can associate with each such transcript a 2-by-2 "transcript matrix," whose entries are the probability that this transcript occurs when Alice and Bob start with a particular pair of inputs. Now, each such transcript has an associated output value. If we sum together all the transcript matrices with output value 0 , we get a 2 -by-2 "protocol matrix," whose entries show the probability that the protocol outputs 0 when Alice and Bob start with a particular pair of inputs.

Now let us consider what constraints we can place on these matrices. Two types of constraints are immediate: (1) the differential privacy conditions on each input linearly constrain each transcript matrix; and (2) the accuracy conditions linearly constrain the protocol matrix. But these constraints alone would not yield any bound better than $\frac{\lambda}{1+\lambda}$, which is achievable in the client-server setting. The key to obtaining better bounds, and our main obstacle, are conditions which capture the constraint that these matrices must actually arise from a protocol between two players. We consider a condition that we call protocol compatibility that essentially captures the fact that if the two parties' inputs are drawn from independent distributions, then they must remain independent even when conditioned on any particular protocol transcript. This post-execution independence has been useful in other works on differential privacy including the work of McGregor et al. [25], as well as in works on secure computation such as the work of Kilian [23].

The protocol-compatibility constraint manifests itself as a non-linear constraint on transcript matrices. Note that there can be an enormous (exponential in communication complexity) number of possible transcript matrices, and we do not want to have to consider such a large space of variables. In particular, we do not want our bounds to depend in any way on the communication complexity or the number of rounds in the protocol. We avoid this by proving a key lemma that shows how to optimally combine the linear differential privacy constraints with 
the non-linear protocol-compatibility constraint to yield a new linear constraint (Lemma 3.6 in Section 3). This combined linear constraint establishes an upper bound on sums of probabilities from a transcript matrix that combine both the upper and lower bounds from the differential privacy constraints. Because these constraints are linear, they immediately give constraints on the protocol matrix, as well. This gives us our linear program.

We analyze the linear programs that arise specifically for the AND and XOR functionalities, and prove that the linear program is not satisfiable when the accuracy $a$ is higher than a certain value. We prove that these bounds are tight by showing that this accuracy can be achieved for both the AND and XOR functionalities. We stress that our technique is more general, and can be applied to other specific functions to obtain potentially stronger, although not necessarily tight, bounds. (As mentioned above, we focus our attention on AND and XOR because every non-trivial Boolean function must contain an embedded AND or XOR function.)

Related Work. In addition to the works mentioned above, several other works have focused on the issue of accuracy and privacy. In the client-server setting (i.e., where only one party owns the entire database), limitations for a wide class of private algorithms were first shown by Dinur and Nissim [9. The optimality of differentially private mechanisms has since been studied in different models such as answering multiple linear queries [17, contingency tables [20], or certain classes of low-sensitivity queries [8]. In a surprising result of Ghosh et al. [14, a simple geometric mechanism (a discrete version of the additive Laplacian mechanism) was shown to be universally optimal for releasing a single count query to Bayesian consumers. Recently, Haitner et al. [16] showed that CDP two-party protocols with accuracy improving upon the information-theoretic bound of McGregor et al. cannot be black-box reduced to random oracles.

In the secure function evaluation model against computationally unbounded semi-honest adversaries, characterization of deterministic Boolean functionalities was completed by Chor and Kushilevitz [7, and for randomized functionalities by 23. These results establish the "all or nothing" nature of two-party computation under information-theoretic reductions. A related question of characterizing complete deterministic functionalities in the computational setting was considered by Harnik et al. 18. Complete classification of randomized functionalities in the computational setting remains an important research problem.

\section{Notation and Definitions}

Standard Notation. We use symbols $\neg, \vee, \wedge$, and $\oplus$ to denote the standard Boolean operations: NOT, OR, AND, and XOR respectively. The set of natural numbers is denoted by $\mathbb{N}$; for $n \in \mathbb{N}$, we write by $[n]$ as shorthand for the set $\{1,2, \ldots, n\}$. The Hamming distance between two strings $x, y \in\{0,1\}^{n}$ is defined as: $|x-y|_{h}=\left|\left\{i \in[n]: x_{i} \neq y_{i}\right\}\right|$, where $x_{i}, y_{i}$ denote the $i$ th bit of $x, y$ respectively. We denote by $e$, the base of the natural logarithm. 
We now recall the definition of $\epsilon$-differential privacy [10] and $(\epsilon, \delta)$-differential privacy [1].

Definition 2.1 ( $\epsilon$-Differential Privacy). We say that a randomized function $M:\{0,1\}^{n} \mapsto \mathcal{R}$, with a finite range $\mathcal{R}$, is an $\epsilon$-differentially-private $(\epsilon-\mathrm{DP})$ mechanism for $\epsilon \geq 0$ if for every $(x, y) \in\{0,1\}^{n} \times\{0,1\}^{n}$ satisfying $\left|x-x^{\prime}\right|_{h}=1$ and every subset $S \subset \mathcal{R}$ we have that over the randomness of $M$ :

$$
\operatorname{Pr}[M(x) \in S] \leq e^{\epsilon} \times \operatorname{Pr}\left[M\left(x^{\prime}\right) \in S\right] .
$$

Definition $2.2((\epsilon, \delta)$-Differential Privacy). We say that a randomized function $M:\{0,1\}^{n} \mapsto \mathcal{R}$, with a finite range $\mathcal{R}$, is an $(\epsilon, \delta)$-differentially-private mechanism for $\epsilon, \delta \geq 0$ if for every $(x, y) \in\{0,1\}^{n} \times\{0,1\}^{n}$ satisfying $\left|x-x^{\prime}\right|_{h}=$ 1 and every subset $S \subset \mathcal{R}$ we have that over the randomness of $M$ :

$$
\operatorname{Pr}[M(x) \in S] \leq e^{\epsilon} \times \operatorname{Pr}\left[M\left(x^{\prime}\right) \in S\right]+\delta .
$$

We next recall the definition of computational differential privacy which captures differentially privacy for polynomial time tests. We work with the weakest definition, namely $\epsilon$-IND-CDP [26]. In the following, $k$ denotes the security parameter, implicitly available to all algorithms; algorithms are assumed to run in time polynomial in $k$ unless stated otherwise.

Definition 2.3 ( $\epsilon$-IND-CDP Privacy). We say that an ensemble $\left\{M_{\kappa}\right\}_{k \in \mathbb{N}}$ of randomized functions $M_{k}:\{0,1\}^{n} \mapsto \mathcal{R}_{k}$ provides $\epsilon$-IND-CDP if there exists a negligible function negl(.) such that for every probabilistic polynomial time distinguisher $A$, for every polynomial $p(\cdot)$, for any adjacent strings $x, x^{\prime} \in\{0,1\}^{n}$ (i.e., $\left|x-x^{\prime}\right|_{h}=1$ ), for every sufficiently large $k \in \mathbb{N}$, and for every advice string $z_{k}$ of size at most $p(k)$, it holds that

$$
\operatorname{Pr}\left[A_{k}\left(M_{k}(x)\right)=1\right] \leq e^{\epsilon} \times \operatorname{Pr}\left[A_{k}\left(M_{k}\left(x^{\prime}\right)\right)=1\right]+\operatorname{negl}(\kappa),
$$

where we write $A_{k}(x)$ for $A\left(1^{k}, z_{k}, x\right)$ and the probability is taken over the randomness of mechanism $M_{k}$ and the distinguisher $A$.

Interactive Setting. Let $\pi:=\langle A, B\rangle$ be a two-party protocol. Let $\operatorname{VIEW}_{\pi}^{A}(x, y)$ be the random variable which, in a random execution of $\pi$ with inputs $x, y$ for $A, B$ respectively, consists of ( $x, R_{A}$, trans), where $R_{A}$ is the randomness used by $A$ and trans is the sequence of messages exchanged between the parties in the sampled execution. For each $x, \operatorname{VIEW}_{\pi}^{A}(x, y)$ is a mechanism over the $y$ 's. Define $\operatorname{VIEW}_{\pi}^{B}(x, y)$ analogously. When dealing with the computational notion, we consider the family of protocols $\left\{\pi_{k}\right\}_{k \in \mathbb{N}}$ and denote the view of $A$ (resp., $B$ ) by $\operatorname{VIEW}_{\pi}^{A}(k, x, y)$ (resp., $\left.\operatorname{VIEW}_{\pi}^{B}(k, x, y)\right)$.

Definition 2.4 (Two-Party Differentially Privacy). We say that a protocol $\pi:=\langle A, B\rangle$ is $\epsilon$-DP (resp., $(\epsilon, \delta)-D P$ ) if the mechanism $\operatorname{VIEW}_{\pi}^{A}(x, y)$ is $\epsilon$-DP (resp., $(\epsilon, \delta)-D P)$ for all values of $x$ and the same holds for $\operatorname{VIEW}_{\pi}^{B}(x, y)$. $A$ family of protocols $\left\{\pi_{k}\right\}_{k \in \mathbb{N}}$ is $\epsilon$-IND-CDP if the mechanism $\operatorname{VIEW}_{\pi}^{A}(k, x, y)$ is $\epsilon$ IND-CDP for all values of $x$ and every sufficiently large $k$, and the same holds for $\operatorname{VIEW}_{\pi}^{B}(k, x, y)$. 
Finally, our measure of accuracy for Boolean functions simply looks at how often a randomized mechanism outputs the correct output bit in the worst case.

Definition 2.5 (Accuracy). The accuracy of a randomized Boolean mechanism $M:\{0,1\}^{n} \mapsto\{0,1\}$ with respect to a Boolean function $f:\{0,1\}^{n} \mapsto\{0,1\}$ is defined as:

$$
\operatorname{Acc}_{\mathrm{f}}(M)=\min _{x}\{\operatorname{Pr}[M(x)=f(x)]\}
$$

where the probability is taken over the randomness of $M$.

The accuracy of a two party protocol $\pi:=\langle A, B\rangle$ w.r.t. to $f:\{0,1\}^{n} \times\{0,1\}^{n} \mapsto$ $\{0,1\}$ is defined as the accuracy of the mechanism $\operatorname{OUT}_{\pi}:\{0,1\}^{n} \times\{0,1\}^{n} \mapsto$ $\{0,1\}$ which returns the (official) output of the protocol in a randomly sampled execution of $\pi$. The accuracy for a family of protocols $\left\{\pi_{k}\right\}_{k \in \mathbb{N}}$ is defined analogously for each $k$.

\section{Geometric Analysis}

It can be shown that AND and XOR gates are embedded on adjacent inputs into any non-trivial Boolean two-party functionality, i.e., any functionality whose output is not fully determined by one side's input (the proof appears in the full version). Therefore, it will be sufficient to analyze AND/XOR gates. A similar claim also appears in [4] but does not guarantee the adjacency of inputs that embed AND/XOR gates. Adjacency is crucial in our case, since otherwise we cannot conclude that the protocol for AND/XOR have the same privacy parameter $\epsilon$.

We then formulate necessary conditions for existence of a differentially private two-party protocol implementing a randomized two-party Boolean functionality (Section 3.1), and use these conditions towards tight analysis of accuracy of AND and XOR gates achievable via differentially private protocols (Sections 3.2 and $[3.3$.

\subsection{Differential Privacy and Protocol Compatibility}

We begin by introducing several definitions pertaining to properties of matrices that describe joint distributions of protocol outcomes as a function of two inputs. For compactness we will use $\lambda=e^{\epsilon}$ without stating it explicitly throughout the section.

Definition 3.1 ( $\epsilon$-DP Matrix). A $2^{n} \times 2^{n}$ matrix $P$ indexed by strings $x, y \in$ $\{0,1\}^{n}$ is $\epsilon$-DP if its elements satisfy the following conditions for all adjacent pairs $x, x^{\prime} \in\{0,1\}^{n}$ and $y, y^{\prime} \in\{0,1\}^{n}$ :

$$
\begin{aligned}
& p_{x y} \leq \lambda \cdot p_{x y^{\prime}}, \\
& p_{x y} \leq \lambda \cdot p_{x^{\prime} y},
\end{aligned}
$$


Definition 3.2 (Protocol-Compatible Matrix). $A 2^{n} \times 2^{n}$ matrix $P$ is protocol compatible if for all $x_{1}, x_{2}, y_{1}, y_{2} \in\{0,1\}^{n}$ it holds that

$$
p_{x_{1} y_{1}} \cdot p_{x_{2} y_{2}}=p_{x_{1} y_{2}} \cdot p_{x_{2} y_{1}}
$$

The next two definitions extend the concepts of differential privacy and protocol compatibility to two-party Boolean functionalities. By convention, we say that a $2^{n} \times 2^{n}$ matrix $P$ represents a randomized Boolean functionality $f$ of two inputs if $p_{x y}=\operatorname{Pr}[f(x, y)=0]$ for all $x, y \in\{0,1\}^{n}$.

Definition 3.3 ( $\epsilon$-DP Functionality). We call $a 2^{n} \times 2^{n}$ matrix $P$ an $\epsilon$-DP functionality if both $P$ and $\mathbf{1}-P$ are $\epsilon$-DP matrices, where $\mathbf{1}$ is the all-ones matrix.

Definition 3.4. A $2^{n} \times 2^{n}$ matrix $P$ is protocol-compatible $\epsilon$-DP functionality if both matrices $P$ and $\mathbf{1}-P$ can be expressed as sums of protocol-compatible $\epsilon$-DP matrices, where $\mathbf{1}$ is the all-ones matrix.

The following theorem establishes necessary conditions for existence of a differentially private two-party protocol for computing a randomized predicate of two $n$-bit inputs.

Theorem 3.5. Let $\pi$ be a randomized $\epsilon$-DP two-party protocol defined over $x, y \in\{0,1\}^{n}$ and $\pi(x, y)$ be the Boolean output of the protocol. Let $P$ be a matrix of probabilities $p_{x y}=\operatorname{Pr}[\pi(x, y)=0]$. Then $P$ is a protocol-compatible $\epsilon$-DP functionality.

Proof. We start by showing that $P$ can be expressed as sums of protocolcompatible $\epsilon$-DP matrices. The proof for $\mathbf{1}-P$ is analogous.

Let $T_{0}$ be the set of all transcripts $\tau$ for which the protocol output is 0 , i.e. $\pi(x, y)=0$. For a fixed $x, y$, let $\tau \leftarrow \pi(x, y)$ denote that event that in a random execution of $\pi$ with inputs $(x, y)$, the transcript is $\tau$. Let $P_{\tau}$ be a $2^{n} \times 2^{n}$ matrix indexed by $n$-bit strings such that $P_{\tau}(x, y)=\operatorname{Pr}[\tau \leftarrow \pi(x, y)]=p_{\tau, x y}$ (say). Then,

$$
p_{x y}=\operatorname{Pr}[\pi(x, y)=0]=\sum_{\tau \in T_{0}} \operatorname{Pr}[\tau \leftarrow \pi(x, y)]=\sum_{\tau \in T_{0}} p_{\tau, x y} .
$$

Therefore, it holds that $P=\sum_{\tau \in T_{0}} P_{\tau}$. It is easy to verify that the matrices $P_{\tau}$ are $\epsilon$-DP matrices. To complete the proof, we now show that each $P_{\tau}$ is protocol-compatible (following [23]).

Let $X$ and $Y$ be independently and uniformly distributed random variables taking values in $\{0,1\}^{n}$. Then, using Bayes' rule we see that for any two strings $x, y, p_{\tau, x y}=\operatorname{Pr}[X=x, Y=y \mid \tau \leftarrow \pi(X, Y)] \cdot \operatorname{Pr}[\tau \leftarrow \pi(X, Y)] / \operatorname{Pr}[X=x, Y=$ $y$ ]. It is well known in communication complexity (e.g., see [25]) that for any twoparty protocol $\pi$, if the inputs $X$ and $Y$ are independent before the execution, then for any transcript $\tau$ of the protocol, $X$ and $Y$ remain independent when conditioned on the transcript being $\tau$. That is, $\operatorname{Pr}[X=x, Y=y \mid \tau \leftarrow \pi(X, Y)]=$ 
$\operatorname{Pr}[X=x \mid \tau \leftarrow \pi(X, Y)] \cdot \operatorname{Pr}[Y=y \mid \tau \leftarrow \pi(X, Y)]$. Using this with our previous relation, we see that

$$
p_{\tau, x y}=p_{x, \tau} \cdot p_{y, \tau} \cdot p_{\tau} \cdot 2^{2 n},
$$

where $p_{x, \tau}=\operatorname{Pr}[X=x \mid \tau \leftarrow \pi(X, Y)] ; p_{y, \tau}$ and $p_{\tau}$ are defined analogously. It then follows that for any distinct $x_{1}, y_{1}, x_{2}, y_{2}$ :

$$
p_{\tau, x_{1} y_{1}} \cdot p_{\tau, x_{2}, y_{2}}=p_{x_{1}, \tau} p_{x_{2}, \tau} p_{y_{1}, \tau} p_{y_{2}, \tau} \cdot p_{\tau}^{2} \cdot 2^{4 n}=p_{\tau, x_{1} y_{2}} \cdot p_{\tau, x_{2} y_{1}} \text {. }
$$

This completes the proof for protocol-compatibility, and hence the theorem.

The following lemma plays a critical role in our analysis, as it replaces a pertranscript quadratic constraint imposed by the protocol-compatibility condition with a system of linear inequalities.

Lemma 3.6. If $P$ is protocol-compatible $\epsilon$-DP functionality, then for all adjacent pairs $x, x^{\prime} \in\{0,1\}^{n}$ and $y, y^{\prime} \in\{0,1\}^{n}$

$$
\begin{aligned}
& p_{x y^{\prime}}+p_{x^{\prime} y} \leq p_{x y} / \lambda+p_{x^{\prime} y^{\prime}} \cdot \lambda, \\
& p_{x y^{\prime}}+p_{x^{\prime} y} \leq p_{x y} \cdot \lambda+p_{x^{\prime} y^{\prime}} / \lambda,
\end{aligned}
$$

and

$$
\begin{aligned}
& p_{x y}+p_{x^{\prime} y^{\prime}} \leq p_{x y^{\prime}} / \lambda+p_{x^{\prime} y} \cdot \lambda, \\
& p_{x y}+p_{x^{\prime} y^{\prime}} \leq p_{x y^{\prime}} \cdot \lambda+p_{x^{\prime} y} / \lambda .
\end{aligned}
$$

Proof. We first verify the statement for protocol-compatible $\epsilon$-DP matrices $Q$. Indeed, by the $\epsilon$-DP condition $q_{x y^{\prime}}, q_{x^{\prime} y} \in\left[q_{x y} / \lambda, q_{x^{\prime} y^{\prime}} \cdot \lambda\right]$ and by protocolcompatible $q_{x y^{\prime}} \cdot q_{x^{\prime} y}=q_{x y} \cdot q_{x^{\prime} y^{\prime}}$. If the product of two reals is fixed, their sum is maximized when they are most apart, which corresponds exactly to the endpoints of the feasible interval for $q_{x y^{\prime}}, q_{x^{\prime} y}$. To formalize this, we observe that by simple algebra, the condition

$$
q_{x y} / \lambda \leq q_{x y^{\prime}} \leq q_{x^{\prime} y^{\prime}} \cdot \lambda
$$

is equivalent to the quadratic inequality

$$
q_{x y^{\prime}}^{2}-\left(q_{x y} / \lambda+q_{x^{\prime} y^{\prime}} \cdot \lambda\right) q_{x y^{\prime}}+q_{x y} \cdot q_{x^{\prime} y^{\prime}} \leq 0,
$$

since all probabilities must be non-negative. Rewriting this inequality and dividing by $q_{x y^{\prime}}$, and using the fact that $q_{x^{\prime} y}=q_{x y} \cdot q_{x^{\prime} y^{\prime}} / q_{x y^{\prime}}$, we obtain the desired bound:

$$
q_{x y^{\prime}}+q_{x^{\prime} y} \leq q_{x y} / \lambda+q_{x^{\prime} y^{\prime}} \cdot \lambda .
$$

Moreover, the bound is linear in all $q_{x y}, q_{x y^{\prime}}, q_{x^{\prime} y}, q_{x^{\prime} y^{\prime}}$ and holds for all protocolcompatible $\epsilon$-DP matrices. Therefore, it would also hold for the sum of these matrices, and thus for protocol-compatible $\epsilon$-DP functionalities. The other bounds follow similarly and this completes the proof. 
Lastly, we introduce the following definition that relaxes the notion of the protocolcompatible $\epsilon$-DP functionality to allow for a (typically small or negligible) fraction of non-private transcripts.

Definition 3.7. $A 2^{n} \times 2^{n}$ matrix $P$ is protocol-compatible $\epsilon$-DP functionality if both matrices $P$ and $\mathbf{1}-P-\Delta$ can be expressed as sums of protocol-compatible $\epsilon$-DP matrices, where $\mathbf{1}$ is the all-ones matrix and all entries of $\Delta$ are between 0 and $\delta$.

An analogue of Theorem 3.5 exists for $(\epsilon, \delta)$-functionalities defined over binary inputs:

Theorem 3.8. Let $\pi$ be a randomized $(\epsilon, \delta)$-DP two-party protocol defined over $x, y \in\{0,1\}$ and $\pi(x, y)$ be the Boolean output of the protocol. Let $P$ be a matrix of probabilities $p_{x y}=\operatorname{Pr}[\pi(x, y)=0]$. Then $P$ is a protocol-compatible $(\epsilon+\sqrt{\delta})$ $D P O(\sqrt{\delta})$-close functionality.

Proof. In the notation of the previous theorem, define the set of "bad" transcripts $B$ as

$$
B=\left\{\tau: \exists \text { adjacent } x, x^{\prime}, y, y^{\prime} \in\{0,1\} \text {, s.t. } P_{\tau}(x, y)>e^{\epsilon+\sqrt{\delta}} P_{\tau}\left(x^{\prime}, y^{\prime}\right)\right\} .
$$

We claim that for all $x, y \in\{0,1\}$, the probability that $\operatorname{Pr}[\tau \in B: \tau \leftarrow \pi(x, y)]<$ $O(\sqrt{\delta})$. Applying Theorem 3.5, it is sufficient to prove the claim.

For any two pairs of adjacent inputs $x, x^{\prime}, y, y^{\prime}$ define

$$
B_{x, x^{\prime}, y, y^{\prime}}=\left\{\tau: P_{\tau}(x, y)>e^{\epsilon+\sqrt{\delta}} P_{\tau}\left(x^{\prime}, y^{\prime}\right)\right\} .
$$

The probability of seeing a transcript from $B_{x, x^{\prime}, y, y^{\prime}}$ on input $(x, y)$ is less than $O(\sqrt{\delta})$, since by the guarantee of $(\epsilon, \delta)$-DP and (1):

$$
\begin{aligned}
\operatorname{Pr}\left[\tau \in B_{x, x^{\prime}, y, y^{\prime}}: \tau \leftarrow \pi(x, y)\right\} & \leq e^{\epsilon} \operatorname{Pr}\left[\tau \in B_{x, x^{\prime}, y, y^{\prime}}: \tau \leftarrow \pi\left(x^{\prime}, y^{\prime}\right)\right\}+\delta \\
& <e^{-\sqrt{\delta} \operatorname{Pr}\left[\tau \in B_{x, x^{\prime}, y, y^{\prime}}: \tau \leftarrow \pi(x, y)\right\}+\delta,}
\end{aligned}
$$

from which a $O(\sqrt{\delta})$ bound on $\operatorname{Pr}\left[\tau \in B_{x, x^{\prime}, y, y^{\prime}}: \tau \leftarrow \pi(x, y)\right\}$ follows immediately.

Applying the $(\epsilon, \delta)$-DP condition again, we find that

$$
\operatorname{Pr}\left[\tau \in B_{x, x^{\prime}, y, y^{\prime}}: \pi\left(x^{\prime \prime}, y^{\prime \prime}\right)\right]=e^{\epsilon} O(\sqrt{\delta})
$$

for all $x^{\prime \prime}, y^{\prime \prime} \in\{0,1\}$. Since the event $B$ is the union of all events $B_{x, x^{\prime}, y, y^{\prime}}$, summing over all pairs of adjacent inputs and assuming that $\epsilon$ is constant, we complete the proof.

The next two sections apply Theorem 3.5 to tight analysis of accuracy of differentially private protocols for computing two Boolean functionalities of two bit inputs: AND and XOR. 


\subsection{Analysis of the AND Functionality}

We first define accuracy of a Boolean functionality for computing the AND of two bit inputs specified as a $2 \times 2$ matrix of probabilities. Recall that by convention, the matrix $P$ consists of elements $p_{x y}$ signifying the probability of obtaining output 0 on inputs $(x, y)$.

Definition 3.9 (AND-Accuracy). Define AND-accuracy of a $2 \times 2$ matrix $\left(\begin{array}{ll}p_{00} & p_{01} \\ p_{10} & p_{11}\end{array}\right)$ as

$$
\operatorname{AND}-\operatorname{Acc}\left(\left(\begin{array}{ll}
p_{00} & p_{01} \\
p_{10} & p_{11}
\end{array}\right)\right)=\min \left(p_{00}, p_{01}, p_{10}, 1-p_{11}\right)
$$

Note that this notion is identical to the accuracy defined in Section 2, We prove the following theorem establishing the maximal accuracies achievable by protocol-compatible and arbitrary $\epsilon$-DP functionalities and, in particular, showing that there is a gap between the two quantities.

Theorem 3.10. For any $\lambda \geq 1$ and a $2 \times 2$ matrix $M$ we have the following:

1. If $M$ is a $\epsilon$-DP functionality, then $\operatorname{AND}-\operatorname{Acc}(M) \leq \frac{\lambda}{1+\lambda}$, where $\lambda=e^{\epsilon}$.

2. If $M$ is a $\epsilon$-DP protocol-compatible functionality, then

$$
\operatorname{AND}-\operatorname{Acc}(M) \leq \frac{\lambda\left(\lambda^{2}+\lambda+2\right)}{(1+\lambda)^{3}}
$$

In both cases the equality can be achieved.

Proof. Let $M=\left(\begin{array}{ll}p_{00} & p_{01} \\ p_{10} & p_{11}\end{array}\right)$ and $a=\operatorname{AND}-\operatorname{Acc}(M)$.

Claim 1. The accuracy condition implies $p_{01} \geq a$ and $1-p_{11} \geq a$. On the other hand, by the $\epsilon$-DP constraint $p_{01} \leq p_{11} \cdot \lambda$. Put together we have $a / \lambda \leq p_{11} \leq$ $1-a$, which implies $a \leq \lambda /(1+\lambda)$.

The following matrix is indeed a $\epsilon$-DP functionality with accuracy $\lambda /(1+\lambda)$ :

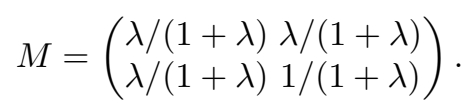

Claim 2. The following conditions relate the probabilities $p_{00}, p_{01}, p_{10}, p_{11}$ to each other and to the accuracy parameter $a$ :

$$
\begin{aligned}
p_{11} & \leq 1-a \\
p_{01}+p_{10} & \geq 2 \cdot a \\
p_{01}+p_{10} & \leq p_{00} / \lambda+p_{11} \cdot \lambda \\
\left(1-p_{01}\right)+\left(1-p_{10}\right) & \leq\left(1-p_{00}\right) \cdot \lambda+\left(1-p_{11}\right) / \lambda .
\end{aligned}
$$

The first two inequalities are implied by the accuracy requirement, the last two by applying Lemma 3.6 to $M$ and $\left(\begin{array}{ccc}1-p_{00} & 1-p_{01} \\ 1-p_{10} & 1-p_{11}\end{array}\right)$. 
By introducing a new variable $q=p_{01}+p_{10}$ and rewriting the above inequalities, we have

$$
\begin{aligned}
& q \geq 2 \cdot a \\
& q \leq p_{00} / \lambda+p_{11} \cdot \lambda \\
& q \geq 2-(\lambda+1 / \lambda)+p_{00} \cdot \lambda+p_{11} / \lambda .
\end{aligned}
$$

Consider the intersection of two lines bounding the half-planes (2) and (4), where $q$ and $p_{00}$ are considered as free variables and $\lambda, a$, and $p_{11}$ are parameters. It is easy to verify that the lines intersect at the point $\left(p_{00}^{*}, q^{*}\right)$, where

$$
p_{00}^{*}=1+1 / \lambda^{2}-2 / \lambda+2 a / \lambda-p_{11} / \lambda^{2} \text { and } q^{*}=2 \cdot a .
$$

The following lemma argues that $\left(p_{00}^{*}, q^{*}\right)$ satisfies (3):

Lemma 3.11. Let $p_{00}^{*}$ be defined as above. Then the following holds:

$$
2 \cdot a \leq p_{00}^{*} / \lambda+p_{11} \cdot \lambda .
$$

Proof. Towards a contradiction, assume that

$$
2 \cdot a>p_{00}^{*} / \lambda+p_{11} \cdot \lambda
$$

Consider two cases:

Case $p_{00} \leq p_{00}^{*}$. Then

$$
q \stackrel{3}{\leq} p_{00} / \lambda+p_{11} \cdot \lambda \leq p_{00}^{*} / \lambda+p_{11} \cdot \lambda \stackrel{\text { 5 }}{<} 2 \cdot a,
$$

contradicting (2).

Case $p_{00}>p_{00}^{*}$. Then

$$
\begin{aligned}
& \left(p_{00}-p_{00}^{*}\right) / \lambda+2 \cdot a \stackrel{5}{>} p_{00} / \lambda+p_{11} \cdot \lambda \stackrel{\text { 3. }}{\geq} q \stackrel{4}{\geq} \\
& 2-(\lambda+1 / \lambda)+p_{00} \cdot \lambda+p_{11} / \lambda=\left(p_{00}-p_{00}^{*}\right) \cdot \lambda+p_{00}^{*} \cdot \lambda+2 \\
& -(\lambda+1 / \lambda)+p_{11} / \lambda \stackrel{\text { def of }}{=} p_{00}^{*}\left(p_{00}-p_{00}^{*}\right) \cdot \lambda+2 \cdot a,
\end{aligned}
$$

which is a contradiction since $\lambda \geq 1$ and $p_{00}>p_{00}^{*}$, concluding the proof of the Lemma.

Finally, by substituting the value of $p_{00}^{*}$ into the statement of Lemma 3.11 and using that $p_{11} \leq 1-a$, we have

$$
\begin{aligned}
2 \cdot a \leq\left(1+1 / \lambda^{2}-2 / \lambda+2 a / \lambda\right. & \left.-p_{11} / \lambda^{2}\right) / \lambda+p_{11} \cdot \lambda \\
& \leq \lambda+1 / \lambda-2 / \lambda^{2}+a \cdot\left(2 / \lambda^{2}-\lambda+1 / \lambda^{3}\right)
\end{aligned}
$$

from which after collecting like terms and simplifying, the claim AND-Acc $(M)=$ $a \leq \lambda\left(\lambda^{2}+\lambda+2\right) /(1+\lambda)^{3}$ follows.

The protocol with optimal accuracy appears in Appendix thus proving tightness of the bound. 
We remark that Claim 2 of Theorem 3.10 also applies to $\delta$-close $\epsilon$-DP protocol compatible functionalities, with the upper bound on the accuracy increasing by $(2+\lambda+1 / \lambda) \delta=O(\delta)$. The proof changes in its application of Lemma 3.6 to $\left(\begin{array}{lll}1-p_{00} & 1-p_{01} \\ 1-p_{10} & 1-p_{11}\end{array}\right)$ that becomes instead $\left(\begin{array}{ccc}1-p_{00}-\delta_{00} & 1-p_{01}-\delta_{01} \\ 1-p_{10}-\delta_{10} & 1-p_{11}-\delta_{11}\end{array}\right)$, where $\delta_{00}, \delta_{01}, \delta_{10}, \delta_{11} \in[0, \delta]$. It is easy to verify that changes in the inequality (4) can be absorbed by reducing the value of $a$ by $(2+\lambda+1 / \lambda) \delta=O(\delta)$.

Maximal accuracies attained by $\epsilon$-DP functionalities and protocol-compatible $\epsilon$-DP functionalities are shown in Figure 1.

\subsection{Analysis of the XOR Functionality}

Recall that we consider the worst-case accuracy of a randomized protocol, i.e., the lowest probability over all inputs of producing a correct answer.

Definition 3.12 (XOR-Accuracy). Define XOR-accuracy of a $2 \times 2$ matrix $\left(\begin{array}{ll}p_{00} & p_{01} \\ p_{10} & p_{11}\end{array}\right)$ as

$$
\operatorname{XOR}-\operatorname{Acc}\left(\left(\begin{array}{cc}
p_{00} & p_{01} \\
p_{10} & p_{11}
\end{array}\right)\right)=\min \left(p_{00}, 1-p_{01}, 1-p_{10}, p_{11}\right) .
$$

Note that this notion is identical to the accuracy defined in Section 2 . The following theorem bounds XOR-accuracy of DP functionalities and protocol-compatible DP functionalities.

Theorem 3.13. For any $\lambda \geq 1$ and a $2 \times 2$ matrix $M$ we have the following:

1. If $M$ is a $\epsilon$-DP functionality, then $\mathrm{XOR}-\operatorname{Acc}(M) \leq \frac{\lambda}{1+\lambda}$.

2. If $M$ is a $\epsilon$-DP protocol-compatible functionality, then $\mathrm{XOR}-\operatorname{Acc}(M) \leq \frac{1+\lambda^{2}}{(1+\lambda)^{2}}$.

In both cases the equality can be achieved.

Proof. Let $M=\left(\begin{array}{cc}p_{00} & p_{01} \\ p_{10} & p_{11}\end{array}\right)$ and $a=\operatorname{AND}-\operatorname{Acc}(M)$.

Claim 1. By the accuracy condition $p_{00} \geq a$ and $1-p_{01} \geq a$. On the other hand, by the $\epsilon$-DP constraint $p_{00} \leq p_{01} \cdot \lambda$. Put together we have $1-a \geq p_{01} \geq$ $p_{00} / \lambda \geq a / \lambda$, which implies $a \leq \lambda /(1+\lambda)$.

The following matrix is indeed a $\epsilon$-DP functionality with accuracy $\lambda /(1+\lambda)$ :

$$
M=\left(\begin{array}{ll}
\lambda /(1+\lambda) & 1 /(1+\lambda) \\
1 /(1+\lambda) & \lambda /(1+\lambda)
\end{array}\right) .
$$

Claim 2. Lemma 3.6 gives the following bounds on the entries of $\epsilon$-DP protocolcompatible matrices:

$$
\begin{aligned}
& p_{00}+p_{11} \leq p_{10} / \lambda+p_{01} \cdot \lambda \\
& p_{00}+p_{11} \leq p_{10} \cdot \lambda+p_{01} / \lambda
\end{aligned}
$$

Summing the inequalities and dividing by two, we have

$$
p_{00}+p_{11} \leq \frac{\lambda^{2}+1}{2 \lambda}\left(p_{10}+p_{01}\right) .
$$


Observe that $\mathrm{XOR}-\operatorname{Acc}(M)=\min \left(p_{00}, 1-p_{01}, 1-p_{10}, p_{11}\right) \leq \min \left(\frac{p_{00}+p_{11}}{2}, 1-\right.$ $\left.\frac{p_{10}+p_{01}}{2}\right)$. Denote $\frac{p_{00}+p_{11}}{2}$ by $x$ and $\frac{p_{10}+p_{01}}{2}$ by $y$, and write

$$
\operatorname{XOR}-\operatorname{Acc}(M)=\min (x, 1-y) \stackrel{6}{\leq} \min \left(x, 1-\frac{2 \lambda}{1+\lambda^{2}} x\right)
$$

which attains its maximal value when $x=1-\frac{2 \lambda}{1+\lambda^{2}} x$. Solving this for $x$ and substituting in the above expression, we prove that

$$
\operatorname{XOR}-\operatorname{Acc}(M) \leq \frac{1+\lambda^{2}}{(1+\lambda)^{2}}
$$

This value of accuracy for computing the XOR functionality is achieved by the randomized response protocol (see Appendix).

\section{One-Way Functions from CDP}

In this paper, we show that one-way functions are implied by the existence of a family of computationally differentially private (CDP) two-party protocols that achieve better accuracy than the bounds proven for DP two-party protocols proven in the previous section. We show this by presenting a more general result: we show that if one-way functions do not exist, then the existence of a family of CDP protocols imply the existence of DP protocols with only negligible loss in accuracy and privacy.

Definition 4.1. An infinite family of two-party protocols $\Pi=\left\{\pi_{k}\right\}$ is defined to be an infinite family of $(\epsilon, \delta=$ negligible)-DP protocols achieving accuracy $a$ for a functionality $F$ if for every constant $c>0$, there exists an infinite sequence of $\pi_{k} \in \Pi$ such that each $\pi_{k}$ is an $\left(\epsilon+k^{-c}, \delta=k^{-c}\right)$-DP protocol with accuracy $a-k^{-c}$ for functionality $F$.

Proof of the following theorem appears in the full version:

Theorem 4.2. Suppose that one-way functions do not exist. Then given any infinite family $\Pi$ of efficient $\epsilon-I N D-C D P$ two-party protocols achieving accuracy a for a functionality $F$, it must be that there is an infinite subfamily $\Pi^{\prime} \subset \Pi$ such that $\Pi^{\prime}$ is an infinite family of $(\epsilon, \delta=$ negligible $)-D P$ protocols achieving accuracy a for the functionality $F$.

Acknowledgements. We thank the anonymous reviewers for their helpful comments.

\section{References}

1. Beaver, D.: Perfect privacy for two-party protocols. In: Feigenbaum, J., Merritt, M. (eds.) Proceedings of DIMACS Workshop on Distributed Computing and Cryptology, vol. 2, pp. 65-77. American Mathematical Society (1989) 
2. Beimel, A., Nissim, K., Omri, E.: Distributed private data analysis: Simultaneously solving how and what. In: Wagner (ed.) 29, pp. 451-468

3. Ben-Or, M., Goldwasser, S., Wigderson, A.: Completeness theorems for non-cryptographic fault-tolerant distributed computation (extended abstract). In: Simon (ed.) 28, pp. 1-10

4. Canetti, R., Kushilevitz, E., Lindell, Y.: On the limitations of universally composable two-party computation without set-up assumptions. In: Biham, E. (ed.) EUROCRYPT 2003. LNCS, vol. 2656, pp. 68-86. Springer, Heidelberg (2003)

5. Chan, T.-H.H., Shi, E., Song, D.: Optimal lower bound for differentially private multi-party aggregation. In: Epstein, L., Ferragina, P. (eds.) ESA 2012. LNCS, vol. 7501, pp. 277-288. Springer, Heidelberg (2012)

6. Chaum, D., Crépeau, C., Damgard, I.: Multiparty unconditionally secure protocols. In: Proceedings of the Twentieth Annual ACM Symposium on Theory of Computing, STOC 1988, pp. 11-19. ACM, New York (1988), http://doi.acm.org/10.1145/62212.62214

7. Chor, B., Kushilevitz, E.: A zero-one law for Boolean privacy. SIAM J. Discrete Math. 4(1), 36-47 (1991)

8. De, A.: Lower bounds in differential privacy. In: Cramer, R. (ed.) TCC 2012. LNCS, vol. 7194, pp. 321-338. Springer, Heidelberg (2012)

9. Dinur, I., Nissim, K.: Revealing information while preserving privacy. In: PODS, pp. 202-210. ACM (2003)

10. Dwork, C.: Differential privacy. In: Bugliesi, M., Preneel, B., Sassone, V., Wegener, I. (eds.) ICALP 2006. Part II, LNCS, vol. 4052, pp. 1-12. Springer, Heidelberg (2006)

11. Dwork, C., Kenthapadi, K., McSherry, F., Mironov, I., Naor, M.: Our data, ourselves: Privacy via distributed noise generation. In: Vaudenay, S. (ed.) EUROCRYPT 2006. LNCS, vol. 4004, pp. 486-503. Springer, Heidelberg (2006)

12. Dwork, C., McSherry, F., Nissim, K., Smith, A.: Calibrating noise to sensitivity in private data analysis. In: Halevi, S., Rabin, T. (eds.) TCC 2006. LNCS, vol. 3876, pp. 265-284. Springer, Heidelberg (2006)

13. Dwork, C., Nissim, K.: Privacy-preserving datamining on vertically partitioned databases. In: Franklin, M. (ed.) CRYPTO 2004. LNCS, vol. 3152, pp. 528-544. Springer, Heidelberg (2004)

14. Ghosh, A., Roughgarden, T., Sundararajan, M.: Universally utility-maximizing privacy mechanisms. In: Mitzenmacher, M. (ed.) Proceedings of the 41st Annual ACM Symposium on Theory of Computing, STOC 2009, pp. 351-360. ACM (2009)

15. Goldreich, O., Micali, S., Wigderson, A.: How to play any mental game or a completeness theorem for protocols with honest majority. In: Aho, A.V. (ed.) STOC, pp. 218-229. ACM (1987)

16. Haitner, I., Omri, E., Zarosim, H.: On the power of random oracles. IACR Cryptology ePrint Archive 2012, 573 (2012)

17. Hardt, M., Talwar, K.: On the geometry of differential privacy. In: Schulman (ed.) [27, pp. 705-714

18. Harnik, D., Naor, M., Reingold, O., Rosen, A.: Completeness in two-party secure computation: A computational view. J. Cryptology 19(4), 521-552 (2006)

19. Ishai, Y., Prabhakaran, M., Sahai, A.: Founding cryptography on oblivious transfer - efficiently. In: Wagner (ed.) 29], pp. 572-591

20. Kasiviswanathan, S.P., Rudelson, M., Smith, A., Ullman, J.: The price of privately releasing contingency tables and the spectra of random matrices with correlated rows. In: Schulman (ed.) [27], pp. 775-784 
21. Kilian, J.: Founding cryptography on oblivious transfer. In: Simon (ed.) [28, pp. $20-31$

22. Kilian, J.: A general completeness theorem for two-party games. In: Koutsougeras, C., Vitter, J.S. (eds.) STOC, pp. 553-560. ACM (1991)

23. Kilian, J.: More general completeness theorems for secure two-party computation. In: STOC, pp. 316-324 (2000)

24. Kushilevitz, E.: Privacy and communication complexity. In: FOCS, pp. 416-421 (1989)

25. McGregor, A., Mironov, I., Pitassi, T., Reingold, O., Talwar, K., Vadhan, S.P.: The limits of two-party differential privacy. In: FOCS, pp. 81-90. IEEE Computer Society (2010)

26. Mironov, I., Pandey, O., Reingold, O., Vadhan, S.: Computational differential privacy. In: Halevi, S. (ed.) CRYPTO 2009. LNCS, vol. 5677, pp. 126-142. Springer, Heidelberg (2009)

27. Schulman, L.J. (ed.): Proceedings of the 42nd ACM Symposium on Theory of Computing, STOC 2010, Cambridge, Massachusetts, USA, June 5-8. ACM (2010)

28. Simon, J. (ed.): Proceedings of the 20th Annual ACM Symposium on Theory of Computing, Chicago, Illinois, USA, May 2-4. ACM (1988)

29. Wagner, D. (ed.): CRYPTO 2008. LNCS, vol. 5157. Springer, Heidelberg (2008)

30. Yao, A.C.C.: Protocols for secure computations (extended abstract). In: 23rd Annual Symposium on Foundations of Computer Science (FOCS 1982), pp. 160-164. IEEE (1982) 